\title{
Broadband X-ray spectrum of intermediate polar V1223 Sgr
}

\author{
M. Revnivtsev ${ }^{1,2}$, A. Lutovinov ${ }^{1}$, V. Suleimanov ${ }^{3}$, R. Sunyaev ${ }^{1,2}$, and V. Zheleznyakov ${ }^{4}$ \\ 1 Space Research Institute, Russian Academy of Sciences, Profsoyuznaya 84/32, 117810 Moscow, Russia \\ e-mail: revnivtsev@hea.iki.rssi.ru \\ 2 Max-Planck-Institute für Astrophysik, Karl-Schwarzschild-Str. 1, 85740 Garching bei München, Germany \\ 3 Kazan State University, Kremlevskaya 18, 420008, Kazan, Russia \\ ${ }^{4}$ Institute of Applied Physics, Ulyanova 46, 603950 Nizhny Novgorod, Russia
}

Received 22 March 2004 / Accepted 29 June 2004

\begin{abstract}
We present the broadband phase averaged spectrum of one of the brightest intermediate polars V1223 Sgr, obtained with INTEGRAL and RXTE observatories (3-100 keV). Good statistical quality of the spectrum in a hard X-ray energy band (INTEGRAL/IBIS and RXTE/HEXTE) allowed us to disentangle contributions of a direct optically thin plasma emission and a reflected component to the spectrum of V1223 Sgr. The obtained measurement of the post-shock temperature of the accreting matter give us the information about the mass of the white dwarf and the inclination of the system.
\end{abstract}

Key words. stars: binaries: general - X-rays: binaries - stars: individual: V1223 Sgr

\section{Introduction}

V1223 Sgr is a very well known binary star that belongs to the class of intermediate polars (IPs) - binary systems with a white dwarf that accrets matter from a Roche lobe filling secondary star (see e.g. comprehensive model and discussion in Beuermann et al. 2004). In the IP systems magnetic field of a white dwarf is strong enough to control the accreting matter at relatively large distances from it and to form a magnetosphere and magnetic funnel (see e.g. Patterson 1994 for a review). The matter falls down to the surface of the white dwarf with almost free fall velocity and is heated in a strong shock to temperatures of the order of $\sim 10 \mathrm{keV}$. Falling matter preferentially comes to the magnetic poles and emits X-rays via an optically thin thermal plasma emission (see e.g. Lamb \& Masters 1979). In spite of this relatively simple scheme the observed emission of IP is much more complicated. First of all its spectrum can contain a set of components with different temperatures (mostly visible at low energies), it often demonstrates an intrinsic photoabsorption, partial covering, different ionization states of an emitting plasma and a reflection from an optically thick relatively medium (see e.g. Warner 1995 for a review). Often the spectrum depends on orbital phase and phase of rotation of the white dwarf (e.g. Norton \& Watson 1989). Different objects of this class were extensively studied by several X-ray observatories over last decades. However, until now there was no study of hard X-ray emission of IP $(E>20 \mathrm{keV})$ except for the case of V709 Cas (de Martino et al. 2001).

Energy band $>20 \mathrm{keV}$ is important because it allows to confidently measure the maximal temperature of the shock in the accretion column of the IP. It is especially important if the X-ray spectrum of IP contains strong reflected component, that distorts the observed continuum at energies $\sim 10-20 \mathrm{keV}$. In the most cases the temperature of the post-shock region of the accretion column of IP is of the order of $\sim 10-40 \mathrm{keV}$ that makes it quite difficult to measure with telescopes working at $0.5-10 \mathrm{keV}$ energy band, like EINSTEIN, ASCA, CHANDRA, XMM. Observations of IP performed with GINGA/LAC (e.g. Ishida 1991; Ishida et al. 1994) practically do not provide statistically significant measurements at $E \sim 20 \mathrm{keV}$, that makes it hard to confidently measure the temperatures higher than $\sim 10-20 \mathrm{keV}$. As the energetics of the X-ray spectrum of IP is dominated by bremsstrahlung emission with maximal temperatures the accuracy of its measurement is of high importance (see. e.g. discussion in Beuermann et al. 2004).

In this work we present results of measurements of the broadband spectrum of the intermediate polar V1223 Sgr with the RXTE and INTEGRAL observatories with an accent on the averaged hard X-ray part of the spectrum.

\section{Data analysis}

The international gamma-ray observatory INTEGRAL was launched by the Russian launcher PROTON from the Baikonur cosmodrome in the high-apogee orbit on October 17, 2002 (Eismont et al. 2003). The payload includes four principal instruments which allow one to carry out simultaneous observations of sources in the X-ray, gamma-ray and optical energy range (Winkler et al. 2003).

In the August-September of 2003 ultra deep ( 2 millionsec) observations of the Galactic center region were performed. The survey of this region with catalog of detected sources was published by Revnivtsev et al. (2004). 


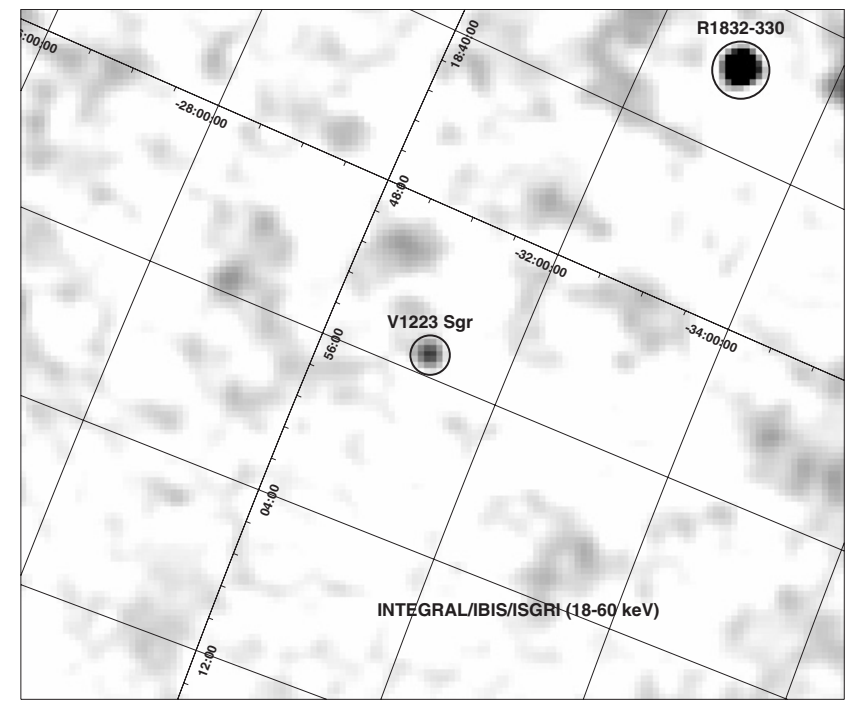

Fig. 1. X-ray map of the region around V1223 Sgr obtained with INTEGRAL/IBIS/ISGRI in the energy band $18-60 \mathrm{keV}$.

The intermediate polar V1223 Sgr was detected during these observations with a high statistical significance in the 18$60 \mathrm{keV}$ energy band.

Due to relatively large distance from the Galactic Center $\left(\sim 16^{\circ}\right), \mathrm{V} 1223 \mathrm{Sgr}$ was outside the field of view of the X-ray monitor JEM-X of the INTEGRAL observatory. On the other hand the source is weak and relatively soft, therefore the sensitivity of the spectrometer SPI (Roques et al. 2003) is not enough to measure the spectrum of the source. Therefore in the paper we will concentrate on the data obtained by IBIS telescope, its upper detector ISGRI (Lebrun et al. 2003). The source was quite far from the center of the field of view of the IBIS telescope, resulting in a strong (factor of $\sim 9.5$ ) reduction of its effective area useful for the measurement of the spectrum of V1223 Sgr.

Data of the IBIS telescope were processed using methods described in Revnivtsev et al. (2004). The image of the region around V1223 Sgr is presented in Fig. 1. For the spectral analysis we used a simple ratio of the flux measured from the source to the fluxes of the Crab nebula in the same energy bands and assumed that the Crab nebula spectrum has the form $\mathrm{d} N(E)=10 E^{-2.1} \mathrm{~d} E$, where $N(E)$ is the number of photons at energy $E$. As the spectrum of V1223 Sgr does not demonstrate very sharp structures such method of the spectral reconstruction is acceptable. Tests of this method on observations of the Crab nebula showed that the resulted spectra can still possess approximately $5 \%$ systematic uncertainties in every energy channel.

In order to compare the obtained results with measurements of other instruments and to accomplish the obtained hard X-ray spectrum of the source with standard X-ray band measurements $(\sim 1-20 \mathrm{keV})$ we used data of the RXTE observatory (Bradt et al. 1993, Rothschild et al. 1998). The PCA and HEXTE spectrometers of this observatory cover the energy range 3-250 $\mathrm{keV}$. Effective areas of these spectrometers are quite large $\sim 6400 \mathrm{~cm}^{2}$ for the PCA at energies $\sim 6-7 \mathrm{keV}$ and $\sim 650 \mathrm{~cm}^{2}$ for each (of two) clusters of HEXTE detectors at energies $\sim 50 \mathrm{keV}$. We quote here effective area of only one HEXTE cluster of detectors because only one HEXTE cluster can observe the source at the same time, the other one observes the background at this time.

Intermediate polar V1223 Sgr was observed by the RXTE observatory 14 times during period 1996-2000. Total usable exposure time of these observation is approximately $114 \mathrm{ks}$.

The data of the RXTE observatory was reduced with the help of standard LHEASOFT/FTOOLS 5.3 package. As the spectrum of IPs does not vary much at energies higher than $>3 \mathrm{keV}$ (see e.g. Beardmore et al. 2000 for the case of V1223 Sgr) for the following analysis we averaged all available data and analyzed only averaged spectrum of V1223 Sgr. For the spectral approximation we used XSPEC package. Normalizations of spectra obtained by different instruments were set free to vary during the fitting procedure. Systematic errors $1 \%$ and $5 \%$ were added to every channel of the RXTE/PCA and INTEGRAL/IBIS spectra, respectively. The resulted spectra of all instruments were rescaled to that of the RXTE/PCA.

\section{Spectral analysis}

First of all we approximated the hard X-ray part of the spectrum of V1223 Sgr obtained with INTEGRAL/IBIS and RXTE/HEXTE (energy band 20-100 keV) with a simplest thermal model - an optically thin thermal bremsstrahlung. The quality of the fit is very good (reduced $\chi^{2} \sim 0.9$ ) and the parameter of the temperature is equal to $k T=29 \pm 2 \mathrm{keV}$. Best fit parameters for separate data of RXTE/HEXTE and INTEGRAL/IBIS are $25.6 \pm 2.5 \mathrm{keV}$ and $29 \pm 5 \mathrm{keV}$, respectively. As the obtained results agree well with each other in our subsequent analysis we will combine data of these two instruments. Obtained value of the temperature parameter is somewhat smaller than that obtained by Beardmore et al. (2000) from GINGA data $(k T=43 \pm 13 \mathrm{keV})$. However if we apply the same model (with the neutral absorption and broad Gaussian line in the energy range $\sim 6-7 \mathrm{keV}$ ) to the data of the RXTE/PCA detector (energy band 3-20 keV) not taking into account harda X-ray data the resulted temperature would be in a very good agreement with that of Beardmore et al. (2000) $-k T=44.5 \pm 1.5 \mathrm{keV}$. Moreover, if we will try to approximate only spectral points in the energy band $10-20 \mathrm{keV}$ we will obtain even higher temperature $-k T=65 \pm 5$. This discrepancy can be understood if we will take into account that the spectrum of V1223 Sgr contains relatively strong reflected (e.g. Basko et al. 1974) component and therefore the model for the spectral approximation should be more complex.

In the work of Beardmore et al. (2000) only GINGA/LAC (energy range 2-37 keV) data were used to describe the source spectrum at energies $>10 \mathrm{keV}$. However, the spectrum of V1223 Sgr obtained by GINGA/LAC practically does not contain statistically significant points at energies higher than $\sim 20 \mathrm{keV}$. In spite of the fact that Beardmore et al. (2000) included reflected component into their spectral approximation it is very hard for their dataset to determine at the same time the temperature of the thermal plasma and the amplitude of the reflection. In our case we have data of INTEGRAL/IBIS and 
Table 1. Best fit parameters for averaged broadband (3-100 keV) spectrum of V1223 Sgr.

\begin{tabular}{l|l}
\hline \hline Parameter & \\
\hline$N_{\mathrm{H}}, 10^{22} \mathrm{~cm}^{-2}$ & $3.3 \pm 0.2$ \\
$k T, \mathrm{keV}$ & $29 \pm 2$ \\
$R=\Omega / 2 \pi$ & $0.35 \pm 0.15$ \\
$E_{\text {Gauss }}$ & $6.63 \pm 0.03$ \\
$\sigma_{\text {Gauss }}$ & $0.37 \pm 0.02$ \\
$E W, \mathrm{eV}$ & $387 \pm 18$ \\
Flux $_{\text {obs }}, 10^{-10} \mathrm{erg} / \mathrm{s} / \mathrm{cm}^{2}$ & $3.76 \pm 0.04^{a}$ \\
Flux $_{\text {corr }}, 10^{-10} \mathrm{erg} / \mathrm{s} / \mathrm{cm}^{2}$ & $4.57 \pm 0.05^{b}$, \\
$\chi^{2} /$ d.o.f. & $76.5 / 89$ \\
\hline &
\end{tabular}

$-R=\Omega / 2 \pi-$ is the parameter of the reflection, solid angle subtended by a reflector.

${ }^{a}$ Observed flux in the energy band $3-100 \mathrm{keV}$, the flux was calculated assuming that the spectrum of the Crab nebula has the following normalization $\mathrm{d} N(E)=10.0 E^{-2.1} \mathrm{~d} E$. If the assumption of the Crab nebula spectrum is different the flux value should be rescaled.

${ }^{b}$ Absorption corrected flux in the energy band $0.5-100 \mathrm{keV}$.

the RXTE/HEXTE (20-100 keV) and can determine this parameters more confidently while fitting the broadband source spectrum.

Approximation of the broadband spectrum (BeppoSAX data) of the another intermediate polar V709 Cas made by de Martino et al. (2001) confirms these arguments. Authors showed that the broadband spectrum of IP should be described by a thermal model with the reflection. Neglecting the X-ray reflection in a spectral modeling result in the overestimation of the temperature of a post-shock plasma in the accretion column of IP.

For the broadband spectral approximation we used a simple analytic model consisting of the bremsstrahlung emission with its reflection from an optically thick cold medium plus the neutral absorption and broad Gaussian line at $\sim 6.5 \mathrm{keV}$. The latter component mimics a set of lines of optically thin plasma and fluorescent Fe line, originating as a result of the reflection. Such spectral model should approximately represent emission of the hot plasma in the post-shock region of V1223 Sgr. The best fit temperature of the bremsstrahlung emission in this case will denote the maximal temperature in the post-shock region of the accretion column of IP. Obtained best fit parameters of the approximation of the combined INTEGRAL and RXTE spectrum of V1223 Sgr are presented in Table 1. The averaged spectrum of V1223 Sgr is shown in Fig. 2. As the value of the plasma temperature strongly correlates with the amplitude of the reflection we also present confidence contours on these values in Fig. 3.

\section{Discussion}

In the previous section we analyzed the broadband phase averaged spectrum of one of the brightest intermediate polar V1223 Sgr. We have shown that even apart from detection of

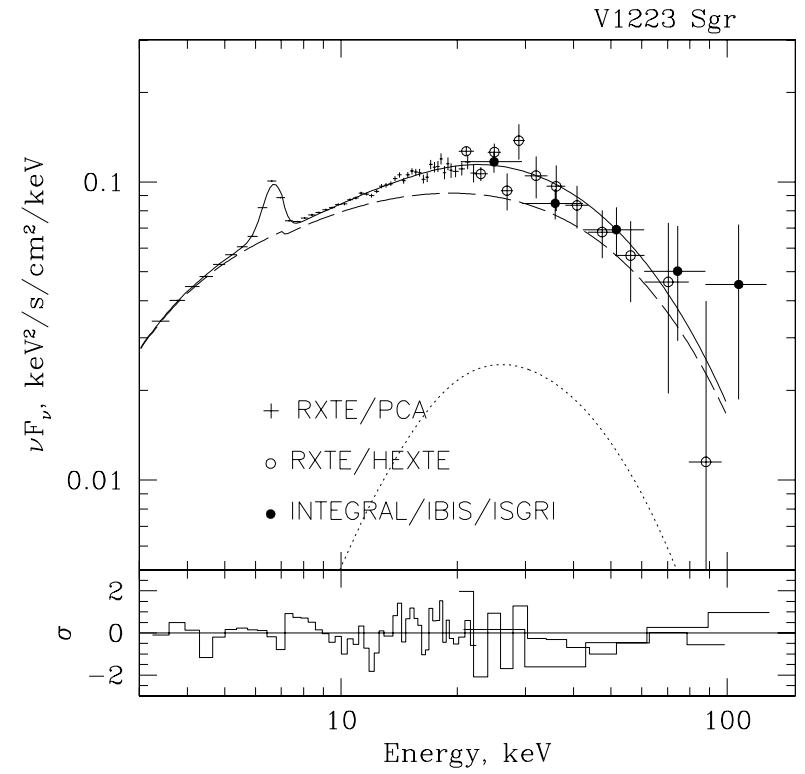

Fig. 2. Averaged spectrum of V1223 Sgr obtained with INTEGRAL and RXTE observatories. Solid curve represents the best fit model. Dashed line shows the emission of the thermal plasma, dotted line the contribution of the reflected component. On the lower panel the difference between data and the model in the units of standard deviations $(\sigma)$ is shown.

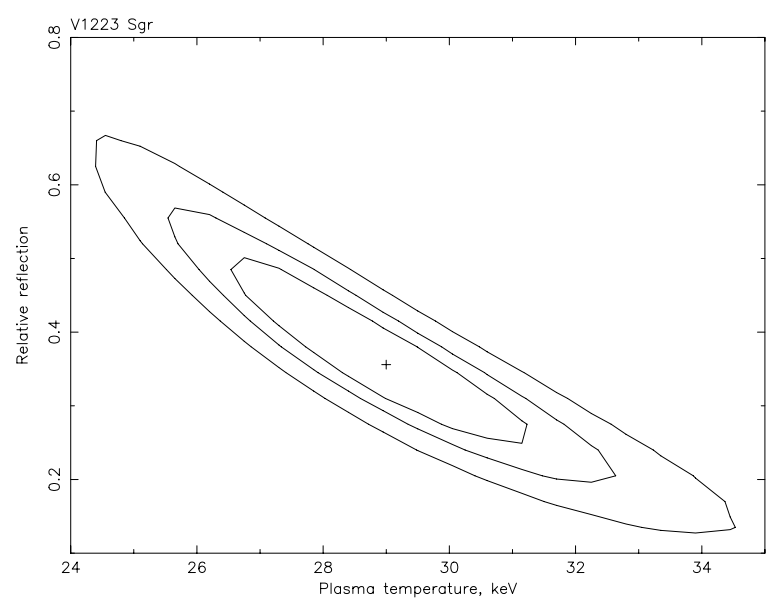

Fig. 3. Confidence contours (1,2 and $3 \sigma)$ of the plasma temperature $(k T)$ and relative reflection amplitude $(R=\Omega / 2 \pi)$.

fluorescent Fe line at energy $\sim 6.4 \mathrm{keV}$ (see Beardmore et al. 2000) there are strong indications on the presence of the reflection component in the spectrum of V1223 Sgr. Because of this component any measurements of the post-shock temperature of the accretion column near the surface of IP that are based on instruments with an effective energy band lower than 20-30 keV are biased towards obtaining higher temperatures. Our spectral modeling for the first time includes hard X-ray energy range (20-100 keV) and provides more confident estimation of the post-shock temperature. Using this value we derive some important parameters of V1223 Sgr.

Current models of a shock in the accretion column of IP assume that the accreted matter is heated up to its virial temperature (see e.g. Aizu 1973; Fabian et al. 1976; 
Lamb \& Masters 1979). The heated plasma produces mainly bremsstrahlung with possible contribution from a cyclotron emission at low accretion rates (see e.g. Fabian et al. 1976; Warner 1995).

Our new broadband measurements of the X-ray spectrum of V1223 Sgr provide us a set of observables with higher confidence than that of previous. They are: 1) the post-shock temperature $T_{\mathrm{S}}=29 \pm 2 \mathrm{keV}, 2$ ) the broadband X-ray flux (absorption corrected) $F_{0.5-100 \mathrm{keV}}=4.6 \times 10^{-10} \mathrm{erg} \mathrm{s}^{-1} \mathrm{~cm}^{-2}$, that in turn gives us the emission measure of one emitting accretion column $E_{\mathrm{m}, 1}=\int N_{\mathrm{e}}^{2} \mathrm{~d} V=1.07 \times 10^{13} 4 \pi D^{2} \mathrm{~cm}^{-3}$, where $N_{\mathrm{e}}$ is the number density of electrons in a hot emitting plasma.

The distance to V1223 Sgr is now known from HST measurement of the parallax of the system, $D=527_{-43}^{+54} \mathrm{pc}$ (Beuermann et al. 2004). Therefore we can evaluate the isotropic luminosity of one accretion column $L_{\mathrm{x}, 1}=$ $4 \pi D^{2} F_{0.5-100 \mathrm{keV}}=(1.5 \pm 0.3) \times 10^{34} \mathrm{erg} \mathrm{s}^{-1}$, and the volume emission measure $E_{\mathrm{m}, 1} \approx 3.4 \times 10^{56} \mathrm{~cm}^{-3}$.

The shock temperature can give the direct evaluation of a white dwarf mass (see e.g. Fabian et al. 1976; Warner 1995)

$T_{\mathrm{s}}=31.9 \frac{M_{\mathrm{WD}}}{M_{\odot}} \frac{10^{9} \mathrm{~cm}}{R_{\mathrm{WD}}} \mathrm{keV}$.

Using an approximation of the white dwarf mass-radius relation from Nauenberg (1972)

$R_{9}=0.78\left[\left(\frac{1.44 M_{\odot}}{M}\right)^{2 / 3}-\left(\frac{M}{1.44 M_{\odot}}\right)^{2 / 3}\right]^{1 / 2}$

where $R_{9}$ is the radius of the white dwarf in the units of $10^{9} \mathrm{~cm}$, we obtain for the mass and radius of the white dwarf in the V1223 Sgr:

$M_{\mathrm{WD}}=0.71 \pm 0.03 M_{\odot}$

$R_{\mathrm{WD}}=(7.8 \pm 0.2) \times 10^{8} \mathrm{~cm}$.

The obtained estimation of the white dwarf mass is slightly smaller than that obtained by Beardmore et al. (2000) because of lower post-shock temperature measured by us and is better consistent with the evaluation of white dwarf mass from optical observations: $M_{\mathrm{WD}}=0.4-0.6 M_{\odot}$ (Penning 1985).

The mass function of the secondary star in the system $\mathrm{V} 1223 \mathrm{Sgr}$ is $f\left(M_{2}\right)=0.0026 \pm 0.0015 M_{\odot}$ (Watts et al. 1985), the mass of the secondary star is $M_{2}=0.4 M_{\odot}$ (Penning 1985). Using these values we can obtain the inclination angle of the system $i=22^{\circ} \pm 5^{\circ}$. If we will assume that the mass of the secondary star is lower, e.g. $M_{2}=0.25 M_{\odot}$ (Beuermann et al. 2004) the estimation of the inclination angle will increase to $i=32^{\circ} \pm 7^{\circ}$.

As the $\mathrm{X}$-ray emission is the major channel of energy losses in the post-shock region we can derive the mass accretion rate $\dot{M}$

$\dot{M} \approx \frac{3}{8} \frac{\mu \mathrm{m}_{\mathrm{H}} 2 L_{\mathrm{x}, 1}}{k T_{\mathrm{s}}}$

where $\mu=0.62$ is the mean molecular weight of the accreting matter. Here factor 2 stands in order to account for two emitting magnetic poles of IP. Substituting measured values we obtain $\dot{M} \approx 2.54 \times 10^{17} \mathrm{~g} \mathrm{~s}^{-1}$.
Using $M_{\mathrm{WD}}=0.71 M_{\odot}, R_{\mathrm{WD}}=7.8 \times 10^{8} \mathrm{~cm}$ and $E_{\mathrm{m}, 1}=$ $4 \pi R_{\mathrm{WD}}^{2} f / 2 h_{\mathrm{s}} N_{\mathrm{e}}^{2} \sim 3.4 \times 10^{56} \mathrm{~cm}^{-3}$, where $f$ is the fraction of the white dwarf surface, occupied by two accretion columns, we can evaluate the height of standing shock $h_{\mathrm{s}}$ and the number density of electrons in the post-shock region $N_{\mathrm{e}}$ following Fabian et al. (1976) and Warner (1995).

$N_{\mathrm{e}}=3.1 \times 10^{13} \dot{M}_{17}\left(\frac{M_{\mathrm{WD}}}{M_{\odot}}\right)^{1 / 2} R_{9}^{-3 / 2} f^{-1}$

$h_{\mathrm{s}}=3.3 \times 10^{4} f \dot{M}_{17}^{-1}\left(\frac{M_{\mathrm{WD}}}{M_{\odot}}\right)^{3 / 2} R_{9}^{1 / 2} \mathrm{~cm}$

where $\dot{M}_{17}$ is the mass accretion rate in units of $10^{17} \mathrm{~g} \mathrm{~s}^{-1}$. We can derive $N_{\mathrm{e}}=9.6 \times 10^{16} f_{-3}^{-1} \mathrm{~cm}^{-3}$ and $h_{\mathrm{s}}=1.1 \times 10^{7} f_{-3} \mathrm{~cm}$, where $f_{-3}=10^{-3} f$ is the fraction of the surface of the white dwarf, occupied by two accretion columns in the units of $10^{-3}$. Note that the derived parameters are similar to those obtained by de Martino et al. (2001) for the intermediate polar V709 Cas.

Acknowledgements. Authors thank Eugene Churazov for developing of algorithms of analysis of data of IBIS telescope and for providing the software. Research has made use of data obtained through the INTEGRAL Science Data Center (ISDC), Versoix, Switzerland, Russian INTEGRAL Science Data Center (RSDC), Moscow, Russia, and High Energy Astrophysics Science Archive Research Center Online Service, provided by the NASA/Goddard Space Flight Center. Work was partially supported by grants of Minpromnauka NSH2083.2003.2 and 40.022.1.1.1103 and program of Russian Academy of Sciences "Non-stationary phenomena in astronomy". M.R. and A.L. thank International Space Science Institute (ISSI), Bern for hospitality and partial support of the work. V.S. acknowledge the support of RFFI grant 02-02-17174 and program of the president of Russian Federation of support of scientific schools NSH-1789.2003.2

\section{References}

Aizu, K. 1973, Prog. Theoret. Phys., 49, 1184

Basko, M., Sunyaev, R., \& Titarchuk, L. 1974, A\&A, 31, 249

Beardmore, A., Osborne, J., \& Hellier, C. 2000, MNRAS, 315, 307

Beuermann, K., Harrison, Th., McArthur, B., Benedict, G., \& Gaensicke, B. 2004, A\&A, 419, 291

Bradt, H., Rothschild, R., \& Swank, J. 1993, A\&AS, 97, 355

de Martino, D., Matt, G., Mukai, K., et al. 2001, A\&A, 377, 499

Eismont, N. A., Ditrikh, A. V., Janin, G., et al. 2003, A\&A, 411, 37

Fabian, A. C., Pringle, J., \& Rees, M. 1976, MNRAS, 175, 43

Ishida, M. 1991, Ph.D. Thesis, Univ. of Tokyo

Ishida, M., Makishima, K., Mukai, K., \& Masai, K. 1994, MNRAS, 266, 367

Lamb, D., \& Masters, A. 1979, ApJ, 234, L117

Lebrun, F., Leray, J. P., Lavocat, P., et al. 2003, A\&A, 411, 141

Nauenberg, M. 1972, ApJ, 175, 417

Norton, A., \& Watson, M. 1989, MNRAS, 237, 853

Patterson, J. 1994, PASP, 106, 209

Penning, W. 1985, ApJ, 289, 300

Revnivtsev, M., Sunyaev, R., Varshalovich, D., et al. 2004, Astron. Lett., 30, 430

Roques, J. P., Schanne, S., von Kienlin, A., et al. 2003, A\&A, 411, 91

Rothschild, R., Blanco, P., Gruber, D., et al. 1998, ApJ, 496, 538

Warner, B. 1995, Cataclysmic variable stars (Cambridge University Press)

Watts, D., Giles, A., Greenhill, J., et al. 1985, MNRAS, 215, 83

Winkler, C., Courvoisier, T. J.-L., Di Cocco, G., et al. 2003, A\&A, 411, L1 